\title{
(+)-CSA Catalyzed Multicomponent Synthesis of 1-[(1,3-Thiazol-2-ylamino)methyl]-2-naphthols and Their Ring-Closure Reaction under Ultrasonic Irradiation
}

\author{
Emel Pelit ${ }^{1}$ and Zuhal Turgut ${ }^{2}$ \\ ${ }^{1}$ Department of Chemistry, Faculty of Art and Sciences, Kirklareli University, Kayali Campus, 39100 Kirklareli, Turkey \\ ${ }^{2}$ Department of Chemistry, Faculty of Art and Sciences, Yildiz Technical University, Davutpaşa Campus, 34210 Istanbul, Turkey \\ Correspondence should be addressed to Emel Pelit; epelit@klu.edu.tr
}

Received 12 February 2016; Accepted 29 March 2016

Academic Editor: Rajeev Sakhuja

Copyright (C) 2016 E. Pelit and Z. Turgut. This is an open access article distributed under the Creative Commons Attribution License, which permits unrestricted use, distribution, and reproduction in any medium, provided the original work is properly cited.

New 1-[(1,3-thiazol-2-ylamino)methyl]-2-naphthols were obtained by condensation of 2-aminothiazole, aromatic aldehydes, and 2 -naphthol in the presence of (+)-camphor-10-sulfonic acid ((+)-CSA) as an effective catalyst under ultrasound-promoted solvent-free conditions. The 1-[(1,3-thiazol-2-ylamino)methyl]-2-naphthol derivatives were converted in ring-closure reaction with formaldehyde to the corresponding naphthoxazine derivatives.

\section{Introduction}

Multicomponent reactions have increasing importance in synthetic organic chemistry because they allow the building of several new bonds in a single step [1-4]. Some of the significant advantages of multicomponent reactions over conventional reactions are a high degree of atomic economy, structural diversity, easier progress of reactions, decreased reaction times, low power consumption, and lack of waste products $[5,6]$.

The Mannich reaction is one of the most important carbon-carbon bond forming reactions in organic chemistry. This reaction is used as a key step in the synthesis of many synthetically and biologically important nitrogen-containing compounds [7-11]. A large number of studies on Mannich-type reactions have been reported under many varied processes in the literature [12-16]. The Mannich reaction has also been performed to achieve stereoselective synthesis of aminonaphthol derivatives in the last decades. These stereoselective compounds have exhibited fairly good enantioselectivity in asymmetric reactions [17-20]. There are some reports on the use of heteroarylamines in Mannichtype reactions and synthesis of 2-amino-1,3-thiazoles in the literature [21-26].

CSA has been demonstrated to be an efficient, nontoxic, ecofriendly, economical, and water soluble catalyst for several reactions such as synthesis of $\beta$-amino carbonyl compounds [27], Friedel-Crafts reactions [28, 29], Meyer-Schuster reactions [30], Friedlander annulations [31], synthesis of $\alpha$ hydroxy and $\alpha$-amino phosphonates [32], synthesis of 1,3,4oxadiazoles [33], synthesis of pseudoglycosides [34], synthesis of coumarins [35], synthesis of spirocyclic compounds [36], and rearrangement of 1,2-dialkynylallyl alcohols [37], and it is widely used in the optical resolution of amines [38].

The development of efficient and environmentally friendly chemical processes has an increasing popularity in organic chemistry. In this context, ultrasound-assisted organic reactions have become an important research area in recent years [39-43]. Ultrasound irradiation is able to activate many organic reactions due to cavitational collapse $[44,45]$. Compared with traditional methods, a broad range of organic syntheses can be successfully performed in higher 
yields and selectivity, shorter reaction times, and milder reaction conditions under ultrasonic irradiation [46-48].

1,3-Oxazine derivatives have shown many biological properties such as analgesic, anticonvulsant, antitubular, antibacterial, antifungal, and anticancer activity [49-56]. Also, in the last decades, these compounds have been used in the treatment of AIDS and Parkinson's disease [57, 58]. Furthermore, 1,3-oxazine derivatives can also be used as an intermediate in the synthesis of $\mathrm{N}$-substituted aminoalcohols [59]. Therefore, several methods for the synthesis of 1,3-oxazine derivatives have been reported [60-66].

Here, we report on the preparation of 1-[(1,3-thiazol2-ylamino)methyl]-2-naphthols catalyzed by (+)-CSA $((+)$ camphor-10-sulfonic acid) under ultrasonic irradiation and solvent-free conditions and then ring-closure reaction of these compounds with formaldehyde under ultrasonic irradiation in THF solvent with short reaction times and good yields.

\section{Experimental}

2.1. Materials and Methods. NMR spectra were determined on a Bruker Avance III-500 MHz NMR. Chemical shifts are given in ppm downfield from $\mathrm{Me}_{4} \mathrm{Si}$ in DMSO- $d_{6}$ or $\mathrm{CDCl}_{3}$ solution. Coupling constants are given in $\mathrm{Hz}$. The FTIR spectra were recorded on a Perkin-Elmer FTIR spectrometer (ATR) and absorption frequencies are reported in $\mathrm{cm}^{-1}$. MS spectra were recorded on a Thermo Elemental X Series ICP-MS. Elemental analyses were measured with Flash EA 1112 Series apparatus and were in good agreement $( \pm 0.2 \%)$ with the calculated values. Ultrasonication was performed in a Bandelin Sonorex Ultrasonic Bath (Super RK) with a frequency of $35 \mathrm{kHz}$ and a power of $230 \mathrm{~W}$. The internal dimensions of the ultrasonic cleaner tank were $240 \times 140$ $\times 100 \mathrm{~mm}$ with liquid holding capacity of $3 \mathrm{~L}$. The reactor was a $100 \mathrm{~mL}$ Pyrex round-bottom flask. The reaction flasks were suspended in the center of the bath, and the addition or removal of water controlled the temperature of the water bath. Melting points were measured on a Gallenkamp melting-point apparatus. Silica gel 60 (Merck) was used for column separation. TLC was conducted on standard conversion aluminum sheets precoated with a $0.2 \mathrm{~mm}$ layer of silica gel. All reagents were commercially available. Anhydrous (+)CSA was purchased from commercial suppliers.

2.2. General Procedure for the Synthesis of 1-[(1,3-Thiazol-2ylamino)methyl]-2-naphthols (1a-n) under Ultrasonic Irradiation. A mixture of (+)-CSA (11.6 mg, $0.05 \mathrm{mmol}), 2$ aminothiazole $(1.00 \mathrm{mmol})$, aromatic aldehyde $(1.00 \mathrm{mmol})$, and 2-naphthol $(1.00 \mathrm{mmol})$ was irradiated with ultrasound of low power (with a frequency of $35 \mathrm{kHz}$ and a nominal power of $230 \mathrm{~W}$ ) at $50^{\circ} \mathrm{C}$ for the period of time indicated in Table 3 and Scheme 3. The reaction flask was located at the maximum energy area in the cleaner and the surface of the reactants was placed slightly lower than the level of the water. The addition or removal of water controlled the temperature of the water bath. After completion of the reaction, as indicated by TLC monitoring, the resultant solid was crystallized from acetone to give products $\mathbf{1 a}-\mathbf{n}$.
1-[Phenyl(1,3-thiazol-2-ylamino)methyl]-2-naphthol (1a). White powder; m.p. 197-199 C (Lit. [23] 196-198 ${ }^{\circ} \mathrm{C}$ ); FTIR (ATR, $\mathrm{cm}^{-1}$ ): 3363, 3131, 3055, 3024, 2971, 1629, 1553, 1514, $1439,1338,1264,1248,1157,1055,1029,949,736 .{ }^{1} \mathrm{H}$ NMR $\delta_{\mathrm{H}}$ $\left(\mathrm{DMSO}-d_{6}, 500 \mathrm{MHz}\right): 6.50(\mathrm{~d}, J=6.00 \mathrm{~Hz}, 1 \mathrm{H}), 7.10-8.36$ $(\mathrm{m}, 13 \mathrm{H}), 9.36(\mathrm{~d}, J=1.50 \mathrm{~Hz}, 1 \mathrm{H}), 12.22$ (br s, $1 \mathrm{H}) .{ }^{13} \mathrm{C}$ NMR $\delta_{\mathrm{C}}\left(\mathrm{DMSO}-d_{6}, 125 \mathrm{MHz}\right): 56.28,107.32,120.21,123.51$, $123.78,123.97,124.73,127.29,127.71,129.07,129.65,130.55$, 136.00, 139.80, 143.96, 150.32, 155.37, 171.06. MS m/z (ESI): 333 $\left(\mathrm{M}^{+}+1\right), 233,215,101,71,57$. Anal. Calc. for $\mathrm{C}_{20} \mathrm{H}_{16} \mathrm{~N}_{2} \mathrm{OS}: \mathrm{C}$, 72.26; H, 4.85; N, 8.43. Found: C, 72.13; H, 4.81; N, 8.40.

1-[4-Methylphenyl(1,3-thiazol-2-ylamino)methyl]-2-naphthol ( $\mathbf{l} \boldsymbol{b}$; Supporting Information Page 2 in Supplementary Material available online at $h t t p: / / d x$.doi.org/10.1155/2016/9315614). White powder; m.p. $163-165^{\circ} \mathrm{C}$; FTIR (ATR, $\mathrm{cm}^{-1}$ ): 3357, $3131,3055,3020,2939,1628,1541,1513,1436,1325,1262$, $1246,1155,1051,1020,953,869,805,782,738 .{ }^{1} \mathrm{H}$ NMR $\delta_{\mathrm{H}}$ (DMSO- $\left.d_{6}, 500 \mathrm{MHz}\right): 2.21$ (s, 3H), 6.59 (br s $\left.1 \mathrm{H}\right), 6.98-7.32$ $(\mathrm{m}, 9 \mathrm{H}), 7.74(\mathrm{~d}, J=8.00 \mathrm{~Hz}, 1 \mathrm{H}), 7.78(\mathrm{~d}, J=8.50 \mathrm{~Hz}, 1 \mathrm{H})$, 7.89 (br s $1 \mathrm{H}), 8.34$ (br s $1 \mathrm{H}), 10.15$ (br s, $1 \mathrm{H}) .{ }^{13} \mathrm{C}$ NMR $\delta_{\mathrm{C}}$ (DMSO- $d_{6}, 125 \mathrm{MHz}$ ): $20.52,53.43,106.62,112.90,118.48$, $119.26,122.30,126.08,128.43,128.54,128.62,129.19,132.12$, $132.75,135.06,138.19,139.55,153.02,168.92$. MS $\mathrm{m} / z$ (ESI): $347\left(\mathrm{M}^{+}+1\right), 247,232,214,189,101,71,57$. Anal. Calc. for $\mathrm{C}_{21} \mathrm{H}_{18} \mathrm{~N}_{2}$ OS: C, 72.80; H, 5.24; N, 8.09. Found: C, 72.76; H, $5.21 ; \mathrm{N}, 8.05$.

1-[4-Bromophenyl(1,3-thiazol-2-ylamino)methyl]-2-naphthol (1c; Supporting Information Page 3). White powder; m.p. $173-175^{\circ} \mathrm{C}$; FTIR (ATR, $\mathrm{cm}^{-1}$ ): 3377, 3113, 3060, 3020, 2966, $1630,1548,1509,1435,1338,1264,1245,1158,1054,1009,946$, $842,805,775,741 .{ }^{1} \mathrm{H}$ NMR $\delta_{\mathrm{H}}\left(\mathrm{DMSO}-d_{6}, 500 \mathrm{MHz}\right): 6.63$ $(\mathrm{d}, J=3.50 \mathrm{~Hz}, 1 \mathrm{H}), 7.00(\mathrm{~d}, J=3.50 \mathrm{~Hz}, 1 \mathrm{H}), 7.04(\mathrm{~d}, J=$ $7.00 \mathrm{~Hz}, 1 \mathrm{H}), 7.16(\mathrm{~d}, J=8.00 \mathrm{~Hz}, 2 \mathrm{H}), 7.22(\mathrm{~d}, J=8.50 \mathrm{~Hz}$, $1 \mathrm{H}), 7.26(\mathrm{~d}, J=7.50 \mathrm{~Hz}, 1 \mathrm{H}), 7.35(\mathrm{~d}, J=5.50 \mathrm{~Hz}, 1 \mathrm{H}), 7.44$ $(\mathrm{d}, J=8.50 \mathrm{~Hz}, 2 \mathrm{H}), 7.77(\mathrm{~d}, J=8.00 \mathrm{~Hz}, 1 \mathrm{H}), 7.80(\mathrm{~d}, J=$ $8.00 \mathrm{~Hz}, 1 \mathrm{H}), 7.84($ br s, $1 \mathrm{H}), 8.38(\mathrm{~d}, J=4.50 \mathrm{~Hz}, 1 \mathrm{H}), 10.20$ (br s, $1 \mathrm{H}) .{ }^{13} \mathrm{C}$ NMR $\delta_{\mathrm{C}}$ (DMSO-d 6 , $125 \mathrm{MHz}$ ): 53.00, 106.99, $118.36,118.65,119.03,122.42,126.28,127.38,128.38,128.53$, $129.53,130.81,132.00,133.49,138.21,142.41,153.03,168.68$. MS $m / z$ (ESI): $412\left(\mathrm{M}^{+}+1\right), 312,232,214,189,101,71,57$. Anal. Calc. for $\mathrm{C}_{20} \mathrm{H}_{15} \mathrm{BrN}_{2} \mathrm{OS}$ : C, 58.40; H, 3.68; N, 6.81. Found: C, 58.25; H, 3.63; N, 6.78.

1-[4-Isopropylphenyl(1,3-thiazol-2-ylamino)methyl]-2-naphthol (1d; Supporting Information Page 4). White powder; m.p. 159-161 ${ }^{\circ}$ C; FTIR (ATR, $\mathrm{cm}^{-1}$ ): 3344, 3118, 3060, 3020, 2957, $1625,1531,1505,1435,1326,1272,1255,1155,1046,1019,954$, $860,817,782,744 .{ }^{1} \mathrm{H}$ NMR (DMSO- $\left.d_{6}, 500 \mathrm{MHz}\right): 1.14$ (d, $J=4.00 \mathrm{~Hz}, 6 \mathrm{H}), 2.79-2.81(\mathrm{~m}, 1 \mathrm{H}), 6.60(\mathrm{~d}, J=3.50 \mathrm{~Hz}$, $1 \mathrm{H}), 6.98-7.35(\mathrm{~m}, 9 \mathrm{H}), 7.76\left(\mathrm{dd}, J_{1}=17.50 \mathrm{~Hz}, J_{2}=7.50 \mathrm{~Hz}\right.$, $2 \mathrm{H}), 7.97(\mathrm{~d}, J=2.00 \mathrm{~Hz}, 1 \mathrm{H}), 8.34$ (br s, $1 \mathrm{H}), 10.13$ (br s, $1 \mathrm{H})$. ${ }^{13} \mathrm{C}$ NMR (DMSO- $d_{6}, 125 \mathrm{MHz}$ ): 23.82, 23.90, 32.95, 53.57, $106.60,114.52,118.48,119.21,122.32,125.89,126.20,128.46$, $128.57,129.16,132.20,133.24,138.24,139.90,146.15,153.00$, 168.88. MS $m / z$ (ESI): $375\left(\mathrm{M}^{+}+1\right), 275,233,215,189,157$, 101, 71, 57. Anal. Calc. for $\mathrm{C}_{23} \mathrm{H}_{22} \mathrm{~N}_{2}$ OS: C, 73.76; H, 5.92; N, 7.48. Found: C, 73.72; H, 5.87; N, 7.45. 
1-[2,4-Dimethylphenyl(1,3-thiazol-2-ylamino)methyl]-2-naphthol (1e; Supporting Information Page 5). White powder; m.p. $171-173^{\circ} \mathrm{C}$; FTIR $\left(\mathrm{ATR}, \mathrm{cm}^{-1}\right): 3343,3125,3062,3000,2956$, 2916, 1625, 1604, 1578, 1528, 1504, 1435, 1365, 1330, 1269, 1242, $1156,1083,1043,946,842,816,745 .{ }^{1} \mathrm{H}$ NMR (DMSO- $d_{6}$, $500 \mathrm{MHz}): 2.12(\mathrm{~s}, 3 \mathrm{H}), 2.20(\mathrm{~s}, 3 \mathrm{H}), 6.53(\mathrm{~d}, J=3.50 \mathrm{~Hz}$, $1 \mathrm{H}), 6.87-6.90(\mathrm{~m}, 2 \mathrm{H}), 6.93(\mathrm{~d}, J=3.50 \mathrm{~Hz}, 2 \mathrm{H}), 7.17-7.23$ $(\mathrm{m}, 3 \mathrm{H}), 7.31(\mathrm{t}, J=7.50 \mathrm{~Hz}, 1 \mathrm{H}), 7.73(\mathrm{~d}, J=9.00 \mathrm{~Hz}, 1 \mathrm{H})$, $7.77(\mathrm{~d}, J=7.50 \mathrm{~Hz}, 1 \mathrm{H}), 8.02(\mathrm{~d}, J=9.00 \mathrm{~Hz}, 1 \mathrm{H}), 8.30(\mathrm{br}$ s, $1 \mathrm{H}), 10.08$ (br s, $1 \mathrm{H}),{ }^{13} \mathrm{C}$ NMR (DMSO- $d_{6}, 125 \mathrm{MHz}$ ): $18.97,20.42,52.95,106.20,117.69,118.42,122.21,125.75,127.34$, $128.46,128.52,129.17,131.14,132.67,135.58,136.04,136.70$, 138.35, 153.32, 168.27. MS $m / z$ (ESI): $361\left(\mathrm{M}^{+}+1\right), 318,274$, 260, 246, 101, 71, 57. Anal. Calc. for $\mathrm{C}_{22} \mathrm{H}_{20} \mathrm{~N}_{2} \mathrm{OS}$ : C, 73.30; H, 5.59; N, 7.77. Found: C, 73.25; H, 5.60; N, 7.74.

1-[(2,4-Dichlorophenyl)(1,3-thiazol-2-ylamino)methyl]-2-naphthol (1f; Supporting Information Page 6). White powder; m.p. $164-166^{\circ} \mathrm{C}$; FTIR $\left(\mathrm{ATR}, \mathrm{cm}^{-1}\right)$ : 3336, 3122, 3072, 3027, 2974, 1624, 1578, 1534, 1515, 1505, 1465, 1434, 1370, 1319, 1268, 1183, 1156, 1082, 1052, 1042, 948, 883, 813, 748. ${ }^{1} \mathrm{H}$ NMR $\left(\right.$ DMSO $\left.d_{6}, 500 \mathrm{MHz}\right): 6.60(\mathrm{~d}, J=3.50 \mathrm{~Hz}, 1 \mathrm{H}), 6.98(\mathrm{~d}, J=$ $3.50 \mathrm{~Hz}, 1 \mathrm{H}), 7.01(\mathrm{~d}, J=7.00 \mathrm{~Hz}, 1 \mathrm{H}), 7.12(\mathrm{~d}, J=8.50 \mathrm{~Hz}, 1 \mathrm{H})$, $7.25(\mathrm{t}, J=7.50 \mathrm{~Hz}, 1 \mathrm{H}), 7.39-7.41(\mathrm{~m}, 2 \mathrm{H}), 7.47(\mathrm{~d}, J=2.00 \mathrm{~Hz}$, $1 \mathrm{H}), 7.66(\mathrm{~d}, J=8.50 \mathrm{~Hz}, 1 \mathrm{H}), 7.75(\mathrm{~d}, J=8.00 \mathrm{~Hz}, 1 \mathrm{H}), 7.79$ $(\mathrm{d}, J=9.00 \mathrm{~Hz}, 1 \mathrm{H}), 8.01(\mathrm{~d}, J=6.50 \mathrm{~Hz}, 1 \mathrm{H}), 8.43(\mathrm{br} \mathrm{s}, 1 \mathrm{H})$, 9.94 (br s, $1 \mathrm{H}$ ). ${ }^{13} \mathrm{C}$ NMR (DMSO- $d_{6}, 125 \mathrm{MHz}$ ): 52.57, 106.81, 116.22 , 118.52, 122.31, 122.73, 126.34, 128.26, 128.47, 129.74, $131.28,131.58,132.70,133.09,138.37,139.27,153.69,167.68$. MS $\mathrm{m} / z$ (ESI): $402\left(\mathrm{M}^{+}+1\right), 317,302,256,157,101,71,57$. Anal. Calc. for $\mathrm{C}_{20} \mathrm{H}_{14} \mathrm{Cl}_{2} \mathrm{~N}_{2} \mathrm{OS}$ : C, 59.86; H, 3.52; N, 6.98. Found: C, 59.84; H, 3.50; N, 6.96.

1-[(2,4-Difluorophenyl)(1,3-thiazol-2-ylamino)methyl]-2-naphthol $(\mathbf{l g})$. White powder; m.p. $165-167^{\circ} \mathrm{C}$; FTIR (ATR, $\mathrm{cm}^{-1}$ ): $3313,3126,3069,3055,3024,2970,1620,1607,1548,1515,1500$, $1434,1371,1347,1261,1235,1159,1139,1083,1053,966,850,815$, 744. ${ }^{1} \mathrm{H}$ NMR (DMSO- $\left.d_{6}, 500 \mathrm{MHz}\right): 6.61(\mathrm{~d}, J=3.50 \mathrm{~Hz}$, $1 \mathrm{H}), 6.98-7.27(\mathrm{~m}, 6 \mathrm{H}), 7.40(\mathrm{~d}, J=6.00 \mathrm{~Hz}, 1 \mathrm{H}), 7.61(\mathrm{~d}, J=$ $5.50 \mathrm{~Hz}, 1 \mathrm{H}), 7.64-8.06(\mathrm{~m}, 3 \mathrm{H}), 8.38(\mathrm{br} \mathrm{s}, 1 \mathrm{H}), 10.08$ (br s, 1H). ${ }^{13} \mathrm{C}$ NMR (DMSO- $d_{6}, 125 \mathrm{MHz}$ ): 48.90, 103.43, 106.88, $110.50,117.23,118.42,122.31,122.88,125.75,126.26,128.29$, $128.53,129.52,130.20,132.15,138.28,153.25,167.98$. MS $\mathrm{m} / \mathrm{z}$ (ESI): $369\left(\mathrm{M}^{+}+1\right), 284,269,256,156,138,101,71,57$. Anal. Calc. for $\mathrm{C}_{20} \mathrm{H}_{14} \mathrm{~F}_{2} \mathrm{~N}_{2} \mathrm{OS}$ : C, 65.20; H, 3.83; N, 7.60. Found: C, 65.18; H, 3.80; N, 7.58.

1-[(3-Phenoxyphenyl)(1,3-thiazol-2-ylamino)methyl]-2-naphthol (1h). White powder; m.p. 162-164 ${ }^{\circ}$ C; FTIR (ATR, $\mathrm{cm}^{-1}$ ): 3356, 3122, 3060, 3006, 2939, 1623, 1579, 1531, 1480, 1437, 1317, $1257,1243,1221,1155,1064,1045,971,866,740 .{ }^{1} \mathrm{H}$ NMR (DMSO- $\left.d_{6}, 500 \mathrm{MHz}\right): 6.62(\mathrm{br} \mathrm{s}, 1 \mathrm{H}), 6.78(\mathrm{~d}, J=6.00 \mathrm{~Hz}$, $1 \mathrm{H}), 6.89(\mathrm{~d}, J=6.00 \mathrm{~Hz}, 2 \mathrm{H}), 7.00(\mathrm{~d}, J=9.50 \mathrm{~Hz}, 3 \mathrm{H}), 7.08$ $(\mathrm{d}, J=9.50 \mathrm{~Hz}, 2 \mathrm{H}), 7.24-7.31(\mathrm{~m}, 6 \mathrm{H}), 7.75-7.79(\mathrm{~m}, 2 \mathrm{H})$, 7.86 (br s, 1H), 8.39 (br s, $1 \mathrm{H}), 10.20($ br s, $1 \mathrm{H}){ }^{13} \mathrm{C} \mathrm{NMR}$ (DMSO- $d_{6}, 125 \mathrm{MHz}$ ): $53.20,106.91,116.32,116.89,117.95$, $118.39,118.89,121.53,122.40,123.06,126.14,128.51,128.60$, $129.45,129.62,129.86,132.05,133.44,138.18,145.38,153.03$, 155.97, 156.67, 168.72. MS $m / z$ (ESI): $425\left(\mathrm{M}^{+}+1\right), 406$,
$362,325,318,274,261,256,202,101,71,57$. Anal. Calc. for $\mathrm{C}_{26} \mathrm{H}_{20} \mathrm{~N}_{2} \mathrm{O}_{2} \mathrm{~S}$ : C, 73.56; H, 4.17; N, 8.28. Found: C, 73.53; H, $4.15 ; \mathrm{N}, 8.25$.

1-[(4-Benzyloxyphenyl)(1,3-thiazol-2-ylamino)methyl]-2-naphthol (1i). White powder; m.p. $153-155^{\circ} \mathrm{C}$; FTIR (ATR, $\mathrm{cm}^{-1}$ ): 3385, 3126, 3057, 3021, 2947, 2862, 1628, 1609, 1579, 1554, 1510, $1459,1441,1338,1266,1251,1240,1175,1157,1120,1054,1038$, 1027, 951, 874, 810, 743. ${ }^{1} \mathrm{H}$ NMR (DMSO- $d_{6}, 500 \mathrm{MHz}$ ): 5.01 (s, $2 \mathrm{H}), 6.59(\mathrm{~d}, J=3.50 \mathrm{~Hz}, 1 \mathrm{H}), 6.89(\mathrm{~d}, J=8.50 \mathrm{~Hz}, 2 \mathrm{H})$, $6.98(\mathrm{~d}, J=3.50 \mathrm{~Hz}, 2 \mathrm{H}), 7.14(\mathrm{~d}, J=8.00 \mathrm{~Hz}, 2 \mathrm{H}), 7.21(\mathrm{~d}, J$ $=9.00 \mathrm{~Hz}, 1 \mathrm{H}), 7.24(\mathrm{~d}, J=7.50 \mathrm{~Hz}, 1 \mathrm{H}), 7.30(\mathrm{~d}, J=7.00 \mathrm{~Hz}$, $1 \mathrm{H}), 7.34-7.37(\mathrm{~m}, 3 \mathrm{H}), 7.41(\mathrm{~d}, J=7.00 \mathrm{~Hz}, 2 \mathrm{H}), 7.74(\mathrm{~d}, J=$ $9.00 \mathrm{~Hz}, 1 \mathrm{H}), 7.78(\mathrm{~d}, J=8.00 \mathrm{~Hz}, 1 \mathrm{H}), 7.90$ (br s, $1 \mathrm{H}), 8.33$ (br s, $1 \mathrm{H}), 10.15$ (br s, $1 \mathrm{H}) .{ }^{13} \mathrm{C}$ NMR (DMSO-d 6 , $125 \mathrm{MHz}$ ): 53.25, 69.09, 114.27, 114.91, 118.45, 119.16, 121.18, 122.31, 124.76, $125.05,127.32,127.62,127.74,128.36,128.45,129.17,134.59$, $137.119,138.23,138.67,139.50,150.55,152.99,156.77,168.86 . \mathrm{MS}$ $\mathrm{m} / z$ (ESI): $439\left(\mathrm{M}^{+}+1\right), 420,377,361,339,326,316,299,256$, 247, 154, 101, 71, 57. Anal. Calc. for $\mathrm{C}_{27} \mathrm{H}_{22} \mathrm{~N}_{2} \mathrm{O}_{2}$ S: C, 73.95; H, 5.06; N, 6.39. Found: C, 73.93; H, 5.05; N, 6.36.

1-[(3-Nitrophenyl)(1,3-thiazol-2-ylamino)methyl]-2-naphthol (1j). White powder; m.p. $183-185^{\circ} \mathrm{C}$, (Lit. [23] 181-183 ${ }^{\circ} \mathrm{C}$ ); FTIR (ATR, $\mathrm{cm}^{-1}$ ): 3387, 3124, 3054, 3020, 2927, 2852, 1628, $1606,1574,1537,1458,1440,1336,1263,1250,1237,1155,1120$, $1056,1041,1025,954,876,810,745 .{ }^{1} \mathrm{H}$ NMR (DMSO- $d_{6}$, $500 \mathrm{MHz}): 6.62(\mathrm{~d}, J=3.50 \mathrm{~Hz}, 1 \mathrm{H}), 6.88-7.78(\mathrm{~m}, 9 \mathrm{H}), 7.87$ (br s, $1 \mathrm{H}), 8.00-8.46(\mathrm{~m}, 3 \mathrm{H}), 10.23$ (br s, $1 \mathrm{H}) .{ }^{13} \mathrm{C}$ NMR (DMSO- $d_{6}, 125 \mathrm{MHz}$ ): $53.12,108.07,118.30,118.52,119.76$, $121.15,122.43,123.05,126.40,128.35,128.55,129.55,130.81$, $132.12,133.10,138.23,138.36,144.00,152.98,168.56$. MS $m / z$ (ESI): $378\left(\mathrm{M}^{+}+1\right), 360,332,270,201,101,71,57$. Anal. Calc. for $\mathrm{C}_{20} \mathrm{H}_{15} \mathrm{~N}_{3} \mathrm{O}_{3} \mathrm{~S}$ : C, 63.65; H, 4.01; N, 11.13. Found: C, 63.53; $\mathrm{H}, 4.03$; N, 11.15.

1-[(1,3-Thiazol-2-ylamino)(thiophen-2-yl)methyl]-2-naphthol (1k; Supporting Information Page 7). White powder; m.p. $155-156^{\circ} \mathrm{C}$; FTIR (ATR, $\mathrm{cm}^{-1}$ ): 3326, 3126, 3091, 3061, 2980, 2908, 2869, 1629, 1550,1514,1436,1372,1340,1266, 1247, $1160,1075,1051,945,870,809,744 .{ }^{1} \mathrm{H}$ NMR (DMSO- $d_{6}$, $500 \mathrm{MHz}): 6.63(\mathrm{~d}, J=3.00 \mathrm{~Hz}, 1 \mathrm{H}), 6.77(\mathrm{br} \mathrm{s}, 1 \mathrm{H}), 6.89(\mathrm{~d}, J$ $=3.00 \mathrm{~Hz}, 1 \mathrm{H}), 7.02(\mathrm{~d}, J=3.00 \mathrm{~Hz}, 1 \mathrm{H}), 7.21-7.27(\mathrm{~m}, 3 \mathrm{H}), 7.32$ $(\mathrm{d}, J=4.50 \mathrm{~Hz}, 1 \mathrm{H}), 7,37(\mathrm{br} \mathrm{s}, 1 \mathrm{H}), 7.77(\mathrm{~d}, J=9.00 \mathrm{~Hz}, 1 \mathrm{H})$, $7.80(\mathrm{~d}, J=8.00 \mathrm{~Hz}, 1 \mathrm{H}), 8.01(\mathrm{br} \mathrm{s}, 1 \mathrm{H}), 8.50($ br s, $1 \mathrm{H}), 10.27$ (br s, $1 \mathrm{H}$ ). ${ }^{13} \mathrm{C}$ NMR (DMSO- $d_{6}, 125 \mathrm{MHz}$ ): 50.81, 107.06, $118.40,118.51,122.42,124.03,124.44,126.11,126.53,128.50$, $129.58,131.99,138.22,147.01,153.02,168.33$. MS m/z (ESI): 339 $\left(\mathrm{M}^{+}+1\right), 318,273,256,239,221,202,101,71,57$. Anal. Calc. for $\mathrm{C}_{18} \mathrm{H}_{14} \mathrm{~N}_{2} \mathrm{OS}_{2}$ : C, 63.88; H, 4.17; N, 8.28. Found: C, 63.85; $\mathrm{H}, 4.15 ; \mathrm{N}, 8.26$.

1-[(1,3-Thiazol-2-ylamino)(thiophen-3-yl)methyl]-2-naphthol (1l). White powder; m.p. $154-156^{\circ} \mathrm{C}$; FTIR (ATR, $\mathrm{cm}^{-1}$ ): $3328,3122,3055,2975,1624,1549,1513,1434,1362,1338,1248$, $1156,1142,1053,949,837,804,741 .{ }^{1} \mathrm{H}$ NMR (DMSO-d ${ }_{6}$, $500 \mathrm{MHz}): 6.60(\mathrm{~d}, J=3.50 \mathrm{~Hz}, 1 \mathrm{H}), 6.86(\mathrm{~d}, J=2.50 \mathrm{~Hz}$, $1 \mathrm{H}), 6.97-7.00(\mathrm{~m}, 2 \mathrm{H}), 7.20-7.26(\mathrm{~m}, 3 \mathrm{H}), 7.35-7.39(\mathrm{~m}$, $2 \mathrm{H}), 7.73-7.78(\mathrm{~m}, 2 \mathrm{H}), 8.01(\mathrm{~d}, J=3.50 \mathrm{~Hz}, 1 \mathrm{H}), 8.48$ (br s, 
$1 \mathrm{H}), 10.23$ (br s, $1 \mathrm{H}) .{ }^{13} \mathrm{C}$ NMR (DMSO- $d_{6}, 125 \mathrm{MHz}$ ): 51.29, $106.68,118.55,118.81,120.51,122.33,125.87,127.04,128.45$, $128.58,129.23,132.03,138.13,143.53,152.92,168.62$. MS $m / z$ (ESI): $339\left(\mathrm{M}^{+}+1\right), 318,273,256,239,221,202,101,71,57$. Anal. Calc. for $\mathrm{C}_{18} \mathrm{H}_{14} \mathrm{~N}_{2} \mathrm{OS}_{2}$ : C, 63.88; H, 4.17; N, 8.28. Found: C, 63.86; H, 4.14; N, 8.26.

1-[5-Methylfuran-2-yl(1,3-thiazol-2-ylamino)methyl]-2-naphthol (Im; Supporting Information Page 8). White powder; m.p. $164-166^{\circ}$ C; FTIR (ATR, $\mathrm{cm}^{-1}$ ): 3368, 3129, 3062, 3054, 3014, 2973, 2950, 1632, 1560, 1542, 1509, 1447, 1373, 1338, 1261, 1240, 1215, 1175, 1154, 1103, 1053, 1020, 953, 863, 817, 785, 752. ${ }^{1} \mathrm{H}$ NMR (DMSO- $\left.d_{6}, 500 \mathrm{MHz}\right): 2.14(\mathrm{~s}, 3 \mathrm{H}), 5.96(\mathrm{~d}, J=$ $3.00 \mathrm{~Hz}, 1 \mathrm{H}), 5.99(\mathrm{~d}, J=3.00 \mathrm{~Hz}, 1 \mathrm{H}), 6.58(\mathrm{~d}, J=3.50 \mathrm{~Hz}$, $1 \mathrm{H})$, 6.97-6.98 (m, 2H), 7.18-7.25 (m, 2H), 7.38 (br s, $1 \mathrm{H})$, $7.73-7.78$ (m, 2H), 8.12 (d, $J=8.00 \mathrm{~Hz}, 2 \mathrm{H}), 8.43$ (br s, $1 \mathrm{H})$, 10.18 (br s, $1 \mathrm{H}) .{ }^{13} \mathrm{C}$ NMR (DMSO- $d_{6}, 125 \mathrm{MHz}$ ): 13.32, 49.14, $106.34,106.67,107.28,116.67,118.48,122.33,123.61,125.97$, $128.39,128.48,129.40,132.35,138.17,150.35,152.65,153.31$, 168.25. MS $m / z$ (ESI): $337\left(\mathrm{M}^{+}+1\right), 318,274,256,237,219$, 195, 101, 57. Anal. Calc. for $\mathrm{C}_{19} \mathrm{H}_{16} \mathrm{~N}_{2} \mathrm{O}_{2} \mathrm{~S}$ : C, 67.84; H, 4.79; $\mathrm{N}, 8.33$. Found: C, 67.86; H, 4.77; N, 8.30.

1-[5-Bromofuran-2-yl(1,3-thiazol-2-ylamino)methyl]-2-naphthol (1n; Supporting Information Page 9). White powder; m.p. 158-160 C; FTIR (ATR, cm ${ }^{-1}$ ): 3366, 3129, 3066, 3024, 2991, 2980, 2915, 1632, 1580, 1542, 1508, 1448, 1373, 1337, 1271, 1258, $1198,1154,1122,1105,1052,1009,921,862,816,787,751 .{ }^{1} \mathrm{H}$ NMR (DMSO- $\left.d_{6}, 500 \mathrm{MHz}\right): 6.20(\mathrm{~d}, J=3.00 \mathrm{~Hz}, 1 \mathrm{H}), 6.46$ $(\mathrm{d}, J=3.50 \mathrm{~Hz}, 1 \mathrm{H}), 6.62(\mathrm{~d}, J=3.50 \mathrm{~Hz}, 1 \mathrm{H}), 7.01(\mathrm{~d}, J=$ $3.50 \mathrm{~Hz}, 1 \mathrm{H}), 7.04(\mathrm{~d}, J=6.50 \mathrm{~Hz}, 1 \mathrm{H}), 7.21(\mathrm{~d}, J=9.00 \mathrm{~Hz}$, 1H), 7.27 (t, $J=7.50 \mathrm{~Hz}, 1 \mathrm{H}), 7.40(\mathrm{t}, J=7.50 \mathrm{~Hz}, 1 \mathrm{H}), 7.77$ $(\mathrm{d}, J=9.00 \mathrm{~Hz}, 1 \mathrm{H}), 7.80(\mathrm{~d}, J=8.00 \mathrm{~Hz}, 1 \mathrm{H}), 8.08(\mathrm{~d}, J=$ $8.50 \mathrm{~Hz}, 1 \mathrm{H}), 8.47$ (d, $J=5.00 \mathrm{~Hz}, 1 \mathrm{H}), 10.24$ (br s, $1 \mathrm{H}) .{ }^{13} \mathrm{C}$ NMR (DMSO- $d_{6}, 125 \mathrm{MHz}$ ): 48.83, 106.99, 109.35, 112.28, $115.64,118.31,119.25,122.41,123.15,126.20,128.36,128.45$, 129.72, 132.18, 138.11, 153.38, 156.95, 168.01. MS m/z (ESI): 402 $\left(\mathrm{M}^{+}+1\right), 384,340,302,284,259,255,138,101,71,57$. Anal. Calc. for $\mathrm{C}_{18} \mathrm{H}_{13} \mathrm{BrN}_{2} \mathrm{O}_{2} \mathrm{~S}$ : C, 53.88; $\mathrm{H}, 3.27 ; \mathrm{N}$, 6.98. Found: C, 53.85; H, 3.25; N, 6.96.

2.3. General Procedure for the Synthesis of the Naphthoxazines (2a-f) under Ultrasonic Irradiation. 1-[(1,3-Thiazol2-ylamino)methyl]-2-naphthols $\mathbf{1 a}, \mathbf{1 c}, \mathbf{1 d}, \mathbf{1 k}, \mathbf{1 m}$, and $\mathbf{1 n}$ $(1 \mathrm{mmol})$ were dissolved in THF $(3 \mathrm{~mL})$ and $35 \%$ aqueous formaldehyde $(1.2 \mathrm{mmol})$ was added. The solution was irradiated with ultrasound of low power (with a frequency of $35 \mathrm{kHz}$ and a nominal power of $230 \mathrm{~W}$ ) at $50^{\circ} \mathrm{C}$ for 3 hours. The reaction flask was located at the maximum energy area in the cleaner and the surface of the reactants was placed slightly lower than the level of the water. The addition or removal of water controlled the temperature of the water bath. Solvent was removed and the residue was dried under reduced pressure. The crude oil was purified by column chromatography eluting with EtOAc/hexane.

1-Phenyl-2-(thiazol-2-yl)-2,3-dihydro-1H-naphtho[1,2-e][1,3]oxazine(2a; Supporting Information Page 10). White powder; m.p. $125-126^{\circ}$ C; FTIR (ATR, $\mathrm{cm}^{-1}$ ): 3126, 3106, 3077, 3057,
$3025,2944,2922,2898,2850,1623,1599,1508,1483,1466$, 1404, 1382, 1312, 1233, 1201, 1125, 1089, 1058, 1000, 969, 915, 816, 745. ${ }^{1} \mathrm{H} \mathrm{NMR}\left(\mathrm{CDCl}_{3}, 500 \mathrm{MHz}\right): 5.11(\mathrm{~d}, J=11.00 \mathrm{~Hz}$, $1 \mathrm{H}), 5.64(\mathrm{~d}, J=11.00 \mathrm{~Hz}, 1 \mathrm{H}), 6.67(\mathrm{~d}, J=3.50 \mathrm{~Hz}, 1 \mathrm{H}), 6.87$ (s, $1 \mathrm{H}), 7.13$ (d, $J=9.00 \mathrm{~Hz}, 1 \mathrm{H}), 7.25(\mathrm{~d}, J=3.50 \mathrm{~Hz}, 1 \mathrm{H})$, $7.29-7.33(\mathrm{~m}, 5 \mathrm{H}), 7.36-7.38(\mathrm{~m}, 2 \mathrm{H}), 7.44(\mathrm{~d}, J=9.00 \mathrm{~Hz}, 1 \mathrm{H})$, $7.73(\mathrm{~d}, J=9.00 \mathrm{~Hz}, 1 \mathrm{H}), 7.76(\mathrm{~d}, J=9.00 \mathrm{~Hz}, 1 \mathrm{H}) .{ }^{13} \mathrm{C} \mathrm{NMR}$ $\left(\mathrm{CDCl}_{3}, 125 \mathrm{MHz}\right): 59.53,73.69,109.44,112.86,118.81,123.05$, $123.79,126.92,128.10,128.55,128.64,128.96,129.21,129.72$, $131.89,139.63,140.90,151.69$. MS $m / z$ (ESI): $345\left(\mathrm{M}^{+}+1\right)$, 260, 232, 215, 175, 102, 71, 57. Anal. Calc. for $\mathrm{C}_{21} \mathrm{H}_{16} \mathrm{~N}_{2} \mathrm{OS}$ : C, 73.23; H, 4.68; N, 8.13. Found: C, 73.20; H, 4.66; N, 8.10.

1-(4-Bromophenyl)-2-(thiazol-2-yl)-2,3-dihydro-1H-naphtho[1, 2-e][1,3]oxazine (2b; Supporting Information Page 11). White powder; m.p. 101-103 ${ }^{\circ}$ C; FTIR (ATR, $\mathrm{cm}^{-1}$ ): 3119, 3062, 2952, 2925, 2853, 1625, 1588, 1508, 1484, 1466, 1434, 1401, 1311, 1268, $1230,1176,1131,1057,1000,968,917,877,812,773,745 .{ }^{1} \mathrm{H}$ $\operatorname{NMR}\left(\mathrm{CDCl}_{3}, 500 \mathrm{MHz}\right): 4.99(\mathrm{~d}, J=11.00 \mathrm{~Hz}, 1 \mathrm{H}), 5.52(\mathrm{dd}$, $\left.J_{1}=11.00 \mathrm{~Hz}, J_{2}=2.00 \mathrm{~Hz}, 1 \mathrm{H}\right), 6.62(\mathrm{~d}, J=3.50 \mathrm{~Hz}, 1 \mathrm{H})$, $6.79(\mathrm{~s}, 1 \mathrm{H}), 7.05(\mathrm{~d}, J=9.00 \mathrm{~Hz}, 1 \mathrm{H}), 7.17(\mathrm{~d}, J=8.50 \mathrm{~Hz}, 1 \mathrm{H})$, $7.22-7.33(\mathrm{~m}, 4 \mathrm{H}), 7.36(\mathrm{~d}, J=8.50 \mathrm{~Hz}, 2 \mathrm{H}), 7.56-7.70(\mathrm{~m}$, $3 \mathrm{H}) .{ }^{13} \mathrm{C} \mathrm{NMR}\left(\mathrm{CDCl}_{3}, 125 \mathrm{MHz}\right): 58.69,73.81,109.67,118.80$, $122.84,123.51,123.94,127.08,128.64,129.24,129.95,130.69$, $131.65,131.78,132.46,139.56,139.91,151.68$. MS m/z (ESI): 424 $\left(\mathrm{M}^{+}+1\right)$, 339, 316, 311, 293, 254, 236, 102, 71, 57. Anal. Calc. for $\mathrm{C}_{21} \mathrm{H}_{15} \mathrm{BrN}_{2} \mathrm{OS}$ : C, 59.58; $\mathrm{H}, 3.57 ; \mathrm{N}, 6.62$. Found: C, $59.55 ; \mathrm{H}, 3.55 ; \mathrm{N}, 6.60$.

1-(4-Isopropylphenyl)-2-(thiazol-2-yl)-2,3-dihydro-1H-naphtho[1,2-e][1,3]oxazine (2c; Supporting Information Page 12). White powder; m.p. $115-117^{\circ}$ C; FTIR (ATR, $\mathrm{cm}^{-1}$ ): 3113, 3091, 3059, 3012, 2961, 2921, 2893, 2869, 1624, 1597, 1505, 1485, 1466, 1432, 1404, 1381, 1311, 1271, 1230, 1177, 1126, 1089, 1050, 1001, 973, 918, 857, 812, 773, 746. ${ }^{1} \mathrm{H}$ NMR $\left(\mathrm{CDCl}_{3}, 500 \mathrm{MHz}\right): 1.22$ $(\mathrm{d}, J=3.50 \mathrm{~Hz}, 6 \mathrm{H}), 2.84-2.92(\mathrm{~m}, 1 \mathrm{H}), 5.13(\mathrm{~d}, J=11.00 \mathrm{~Hz}$, $1 \mathrm{H}), 5.65(\mathrm{~d}, J=11.00 \mathrm{~Hz}, 1 \mathrm{H}), 6.66(\mathrm{~d}, J=7.00 \mathrm{~Hz}, 1 \mathrm{H}), 6.82$ $(\mathrm{s}, 1 \mathrm{H}), 7.12(\mathrm{~d}, J=9.00 \mathrm{~Hz}, 1 \mathrm{H}), 7.16(\mathrm{~d}, J=8.00 \mathrm{~Hz}, 1 \mathrm{H})$, $7.25(\mathrm{~d}, J=3.50 \mathrm{~Hz}, 1 \mathrm{H}), 7.27(\mathrm{~d}, J=8.00 \mathrm{~Hz}, 2 \mathrm{H}), 7.31$ (d, $J=$ $8.00 \mathrm{~Hz}, 1 \mathrm{H}), 7.34(\mathrm{~d}, J=7.00 \mathrm{~Hz}, 1 \mathrm{H}), 7.48(\mathrm{~d}, J=8.00 \mathrm{~Hz}$, $1 \mathrm{H}), 7.72(\mathrm{~d}, J=9.00 \mathrm{~Hz}, 1 \mathrm{H}), 7.76(\mathrm{~d}, J=8.00 \mathrm{~Hz}, 1 \mathrm{H}) .{ }^{13} \mathrm{C}$ $\mathrm{NMR}\left(\mathrm{CDCl}_{3}, 125 \mathrm{MHz}\right): 23.90,23.93,33.77,59.46,73.64$, $109.33,113.16,118.81,123.13,123.73,126.67,126.87,128.51$, $128.84,129.18,129.60,131.93,138.24,139.62,148.73,151.62 . M S$ $m / z$ (ESI): $387\left(\mathrm{M}^{+}+1\right), 318,302,274,256,237,218,202,102$, 71, 57. Anal. Calc. for $\mathrm{C}_{24} \mathrm{H}_{22} \mathrm{~N}_{2}$ OS: C, 74.58; H, 5.74; N, 7.25. Found: C, 74.56; H, 5.76; N, 7.23.

2-(Thiazol-2-yl)-1-(thiophen-2-yl)-2,3-dihydro-1H-naphtho[1, 2-e][1,3]oxazine $(\mathbf{2 d})$. White powder; m.p. 117-118 ${ }^{\circ} \mathrm{C}$; FTIR $\left(\right.$ ATR, $\left.\mathrm{cm}^{-1}\right)$ : 3107, 3091, 3072, 3057, 2961, 2922, 2898, 2850, $1623,1598,1505,1485,1467,1405,1376,1361,1312,1230,1169$, 1116, 1087, 1058, 1003, 967, 816, 748, 706. ${ }^{1} \mathrm{H} \mathrm{NMR}\left(\mathrm{CDCl}_{3}\right.$, $500 \mathrm{MHz}): 5.33(\mathrm{~d}, J=11.00 \mathrm{~Hz}, 1 \mathrm{H}), 5.68\left(\mathrm{dd}, J_{1}=11.00 \mathrm{~Hz}\right.$, $\left.J_{2}=2.00 \mathrm{~Hz}, 1 \mathrm{H}\right), 6.69(\mathrm{~d}, J=3.50 \mathrm{~Hz}, 1 \mathrm{H}), 6.82(\mathrm{~d}, J=$ $3.50 \mathrm{~Hz}, 1 \mathrm{H}), 6.87\left(\mathrm{dd}, J_{1}=9.00 \mathrm{~Hz}, J_{2}=3.50 \mathrm{~Hz}, 1 \mathrm{H}\right), 7.02$ $(\mathrm{s}, 1 \mathrm{H}), 7.10(\mathrm{~d}, J=9.00 \mathrm{~Hz}, 1 \mathrm{H}), 7.25(\mathrm{~d}, J=3.50 \mathrm{~Hz}, 1 \mathrm{H})$, $7.30\left(\mathrm{dd}, J_{1}=8.50 \mathrm{~Hz}, J_{2}=1.50 \mathrm{~Hz}, 1 \mathrm{H}\right), 7.32-7.40(\mathrm{~m}, 2 \mathrm{H})$, $7.63(\mathrm{~d}, J=8.50 \mathrm{~Hz}, 1 \mathrm{H}), 7.72(\mathrm{~d}, J=9.00 \mathrm{~Hz}, 1 \mathrm{H}), 7.75(\mathrm{~d}, J$ 


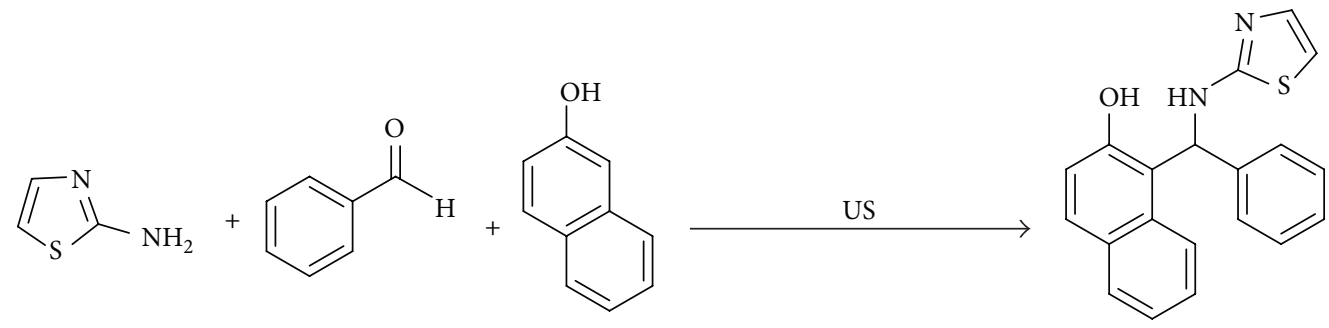

SCHEme 1: One-pot three-component Mannich reaction.

$=7.50 \mathrm{~Hz}, 1 \mathrm{H}) .{ }^{13} \mathrm{C} \mathrm{NMR}\left(\mathrm{CDCl}_{3}, 125 \mathrm{MHz}\right): 55.09,73.83$, $109.71,118.75,122.84,123.88,126.25,126.71,127.02,128.41$, $128.55,129.25,129.97,131.67,139.62,144.46,151.11$. MS $m / z$ (ESI): $351\left(\mathrm{M}^{+}+1\right), 323,268,266,238,220,182,101,71$, 57. Anal. Calc. for $\mathrm{C}_{19} \mathrm{H}_{14} \mathrm{~N}_{2} \mathrm{OS}_{2}$ : C, 65.12; H, 4.03; N, 7.99. Found: C, 65.15; H, 4.05; N, 7.97.

1-(5-Methylfuran-2-yl)-2-(thiazol-2-yl)-2,3-dihydro-1H-naphtho[1,2-e][1,3]oxazine (2e; Supporting Information Page 13). White powder; m.p. $126-128^{\circ} \mathrm{C}$; FTIR (ATR, $\mathrm{cm}^{-1}$ ): 3111 , 3072, 3057, 3025, 2930, 2913, 2893, 2855, 1623, 1599, 1508, $1466,1432,1402,1375,1314,1230,1129,1057,998,969,913,809$, 747, 700. ${ }^{1} \mathrm{H}$ NMR $\left(\mathrm{CDCl}_{3}, 500 \mathrm{MHz}\right): 2.43$ (s, 3H), 5.36 (d, $J$ $=11.00 \mathrm{~Hz}, 1 \mathrm{H}), 5.68\left(\mathrm{dd}, J_{1}=11.00 \mathrm{~Hz}, J_{2}=1.50 \mathrm{~Hz}, 1 \mathrm{H}\right), 6.51$ $\left(\mathrm{dd}, J_{1}=3.50 \mathrm{~Hz}, J_{2}=1.00 \mathrm{~Hz}, 1 \mathrm{H}\right), 6.57\left(\mathrm{dd}, J_{1}=3.50 \mathrm{~Hz}, J_{2}\right.$ $=1.00 \mathrm{~Hz}, 1 \mathrm{H}), 6.67(\mathrm{~d}, J=3.50 \mathrm{~Hz}, 1 \mathrm{H}), 6,90(\mathrm{~s}, 1 \mathrm{H}), 7.09$ (d, $J=8.50 \mathrm{~Hz}, 1 \mathrm{H}), 7.24(\mathrm{~d}, J=3.50 \mathrm{~Hz}, 1 \mathrm{H}), 7.30-7.41(\mathrm{~m}, 2 \mathrm{H})$, $7.67(\mathrm{~d}, J=8.50 \mathrm{~Hz}, 1 \mathrm{H}), 7.71(\mathrm{~d}, J=9.00 \mathrm{~Hz}, 1 \mathrm{H}), 7.74(\mathrm{~d}, J=$ $7.50 \mathrm{~Hz}, 1 \mathrm{H}) .{ }^{13} \mathrm{C} \mathrm{NMR}\left(\mathrm{CDCl}_{3}, 125 \mathrm{MHz}\right): 15.45,55.35,73.72$, $109.57,118.74,122.90,123.83,124.77,126.96,128.38,128.51$, $129.21,129.86,131.71,139.61,140.89,141.75,151.11$. MS $m / z$ (ESI): $349\left(\mathrm{M}^{+}+1\right), 306,264,236,218,180,101,71,57$. Anal. Calc. for $\mathrm{C}_{20} \mathrm{H}_{16} \mathrm{~N}_{2} \mathrm{O}_{2} \mathrm{~S}$ : C, 68.94; $\mathrm{H}, 4.63 ; \mathrm{N}, 8.04$. Found: C, 68.96; H, 4.65; N, 8.02.

1-(5-Bromofuran-2-yl)-2-(thiazol-2-yl)-2,3-dihydro-1H-naphtho[1,2-e][1,3] oxazine (2f). White powder; m.p. $105-107^{\circ} \mathrm{C}$; FTIR (ATR, $\mathrm{cm}^{-1}$ ): 3120, 3065, 2997, 2928, 1625, 1600, 1507, 1467, 1434, 1352, 1314, 1230, 1202, 1164, 1122, 1058, 1000, 976, 950, 908, 811, 782, 728. ${ }^{1} \mathrm{H}$ NMR $\left(\mathrm{CDCl}_{3}, 500 \mathrm{MHz}\right): 5.20$ (d, $J=11.00 \mathrm{~Hz}, 1 \mathrm{H}), 5.58\left(\mathrm{dd}, J_{1}=11.00 \mathrm{~Hz}, J_{2}=1.50 \mathrm{~Hz}, 1 \mathrm{H}\right)$, $5.89(\mathrm{~d}, J=3.00 \mathrm{~Hz}, 1 \mathrm{H}), 6.10(\mathrm{~d}, J=3.00 \mathrm{~Hz}, 1 \mathrm{H}), 6.57(\mathrm{~d}, J$ $=3.50 \mathrm{~Hz}, 1 \mathrm{H}), 6.75(\mathrm{~s}, 1 \mathrm{H}), 6.97(\mathrm{~d}, J=9.00 \mathrm{~Hz}, 1 \mathrm{H}), 7.12(\mathrm{~d}$, $J=3.50 \mathrm{~Hz}, 1 \mathrm{H}), 7.20-7.31(\mathrm{~m}, 2 \mathrm{H}), 7.46(\mathrm{~d}, J=8.50 \mathrm{~Hz}, 1 \mathrm{H})$, $7.60(\mathrm{~d}, J=9.00 \mathrm{~Hz}, 1 \mathrm{H}), 7.63(\mathrm{~d}, J=8.50 \mathrm{~Hz}, 1 \mathrm{H}) .{ }^{13} \mathrm{C} \mathrm{NMR}$ $\left(\mathrm{CDCl}_{3}, 125 \mathrm{MHz}\right): 53.61,74.13,110.14,112.26,114.00,118.77$, $122.28,122.77,123.01,124.01,127.16,128.65,129.28,130.21$, 131.52, 139.48, 151.67. MS $m / z$ (ESI): $414\left(\mathrm{M}^{+}+1\right), 329,306$, 301, 283, 236, 102, 71, 57. Anal. Calc. for $\mathrm{C}_{19} \mathrm{H}_{13} \mathrm{BrN}_{2} \mathrm{O}_{2} \mathrm{~S}$ : C, 55.22; H, 3.17; N, 6.78. Found: C, 55.20; H, 3.15; N, 6.80.

\section{Results and Discussion}

$\mathrm{Li}$ and Mao reported that the reaction of 2-aminothiazole, 2naphthol, and benzaldehyde under solvent-free conditions at $120^{\circ} \mathrm{C}$ gave the desired product in $6 \mathrm{~h}$ (Lit. [23]). We decided to perform this reaction under ultrasonic irradiation to get a
TABLE 1: Screening of catalysts for the three-component reaction of 2-aminothiazole, benzaldehyde, and 2-naphthol.

\begin{tabular}{lcccc}
\hline Catalyst (mol\%) & Solvent & $\begin{array}{c}\text { Temperature } \\
\left({ }^{\circ} \mathrm{C}\right)\end{array}$ & $\begin{array}{c}\text { Time } \\
(\mathrm{min})\end{array}$ & $\begin{array}{c}\text { Yield } \\
(\%)^{\mathrm{a}}\end{array}$ \\
\hline- & - & 25 & 120 & 65 \\
- & - & 50 & 120 & 74 \\
$\mathrm{VB1}(10)$ & - & 50 & 60 & 66 \\
$\mathrm{Sc}(\mathrm{OTf})_{3}(10)$ & - & 50 & 60 & 70 \\
$\mathrm{Bi}(\mathrm{OTf})_{3}(10)$ & - & 50 & 60 & 67 \\
$\mathrm{Yb}(\mathrm{OTf})_{3}(10)$ & - & 50 & 60 & 73 \\
$(+)-\mathrm{CSA}(10)$ & - & 50 & 60 & 87 \\
- & IL1 & 50 & 60 & 32 \\
- & IL2 & 50 & 60 & 34 \\
- & IL3 & 50 & 60 & 40 \\
\hline
\end{tabular}

${ }^{\mathrm{a}}$ Isolated yield.

IL1: 1-benzyl-3-methylimidazoliumchloride.

IL2: 1-ethyl-2,3-dimethylimidazoliumtetrafluoroborate

IL3: 8-ethyl-1,8-diazabicyclo[5,4,0]-7-undecenium trifluoromethanesulfonate.

shorter reaction time, milder reaction conditions, and higher yield.

Initially, the three-component Mannich reaction of benzaldehyde (1.00 mmol), 2-naphthol (1.00 mmol), and 2-aminothiazole $(1.00 \mathrm{mmol})$ was examined under ultrasonic irradiation without a catalyst at room temperature (Scheme 1). The reaction was completed in 120 minutes with a yield of $65 \%$. When the temperature was increased from room temperature to $50^{\circ} \mathrm{C}$, the yield of product increased. In recent years, the field of greener catalysts such as reusable lanthanide triflates, organocatalysts, biodegradable reagents, and ionic liquids has an increasing importance in synthetic organic chemistry [67-70]. In order to observe the effect of several greener catalysts and ionic liquids such as $\mathrm{Bi}(\mathrm{OTf})_{3}$, $\mathrm{Sc}(\mathrm{OTf})_{3}, \mathrm{Yb}(\mathrm{OTf})_{3}, \mathrm{VB1}$ (thiamine hydrochloride), (+)-CSA (camphor-10-sulfonic acid), 1-benzyl-3-methylimidazoliumchloride (IL1), 1-ethyl-2,3-dimethylimidazoliumtetrafluoroborate (IL2), and 8-ethyl-1,8-diazabicyclo[5,4,0]-7-undecenium trifluoromethanesulfonate (IL3), they were examined under ultrasound irradiation (Table 1 ) at $50^{\circ} \mathrm{C}$. As a result, (+)-CSA was found to be the most effective catalyst at $50^{\circ} \mathrm{C}$ for this reaction.

Next, in order to observe the effect of the amount of $(+)$ CSA on the reaction, we also performed the experiments 


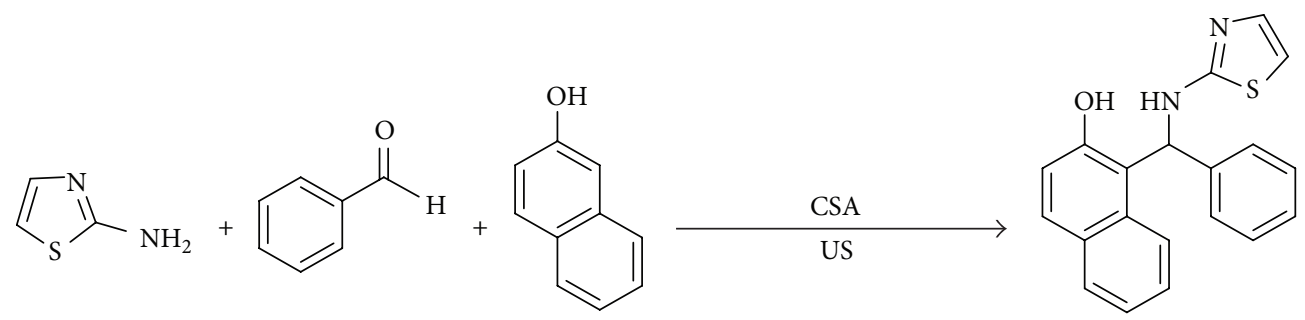

SCHEMe 2

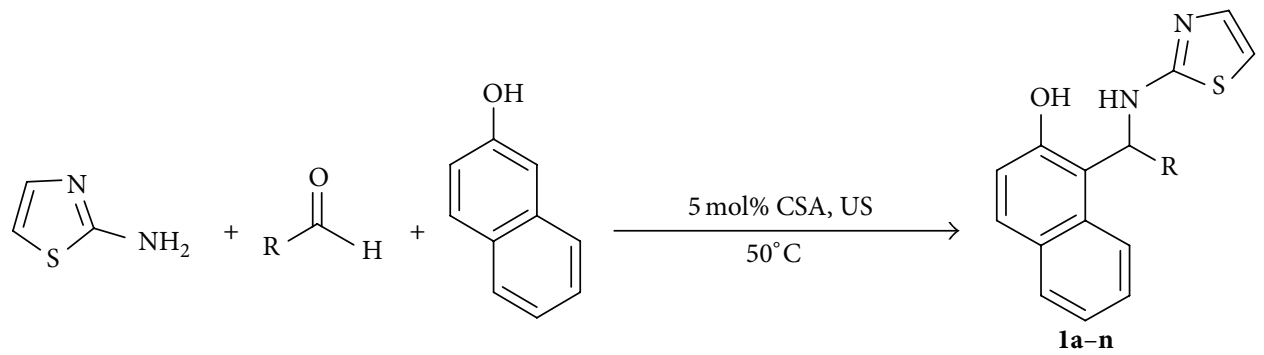

Scheme 3: (+)-CSA catalyzed one-pot three-component Mannich reaction under US conditions.

TABLE 2: Optimization of catalyst loading in the Mannich-type reaction of 2-aminothiazole with benzaldehyde and 2-naphthol using (+)-CSA (see Scheme 2).

\begin{tabular}{lccc}
\hline CSA (mol\%) & Temperature $\left({ }^{\circ} \mathrm{C}\right)$ & Time $(\min )$ & Yield $(\%)^{\mathrm{a}}$ \\
\hline 10 & 25 & 60 & 81 \\
10 & 50 & 30 & 87 \\
15 & 50 & 30 & 84 \\
20 & 50 & 30 & 79 \\
5 & 50 & 30 & 90 \\
\hline
\end{tabular}

${ }^{\mathrm{a}}$ Isolated yield.

using different amounts of catalyst (Table 2 and Scheme 2). The best result was obtained by carrying out the reaction using $5 \mathrm{~mol} \%$ of $(+)-\mathrm{CSA}$ at $50^{\circ} \mathrm{C}$ under solvent-free conditions (Table 2 and Scheme 2).

With these optimal reaction conditions, we then examined a variety of aromatic and heteroaromatic aldehydes in ultrasound-promoted catalytic Mannich-type reactions. As shown in Table 3 and Scheme 3, the one-pot three-component reactions work well with a variety of aromatic and heteroaromatic aldehydes, and the desired compounds were obtained in good yields. However, the aryl aldehydes which contain electron-withdrawing groups gave the desired products in lower yields. On the other hand, reactions with pyridine-2-carbaldehyde and pyridine-4-carbaldehyde did not give the desired product.

The structures of the newly generated compounds have been confirmed by Fourier transform-infrared (FTIR), mass, and NMR techniques. The characteristic absorption bands of $\mathrm{OH}$ and $\mathrm{NH}$ bands were observed at $3326-3385 \mathrm{~cm}^{-1}$ in
TABLE 3: (+)-CSA catalyzed one-pot three-component Mannich reaction of 2-naphthol with 2-aminothiazole and aromatic aldehydes (see Scheme 3).

\begin{tabular}{lccc}
\hline Product & R & Time (min) & Yield (\%) $^{\mathbf{a}}$ \\
\hline $\mathbf{l a}$ & Phenyl & 30 & 90 \\
$\mathbf{l b}$ & 4-Methylphenyl & 30 & 76 \\
$\mathbf{l c}$ & 4-Bromophenyl & 45 & 70 \\
$\mathbf{1 d}$ & 4-Isopropylphenyl & 30 & 87 \\
$\mathbf{l e}$ & 2,4-Dimethylphenyl & 30 & 74 \\
$\mathbf{l f}$ & 2,4-Dichlorophenyl & 45 & 72 \\
$\mathbf{l g}$ & 2,4-Difluorophenyl & 45 & 75 \\
$\mathbf{l h}$ & 3-Phenoxyphenyl & 30 & 83 \\
$\mathbf{l i}$ & 4-Benzyloxyphenyl & 30 & 86 \\
$\mathbf{1 j}$ & 3-Nitrophenyl & 30 & 68 \\
$\mathbf{l k}$ & Thiophen-2-yl & 30 & 75 \\
$\mathbf{l l}$ & Thiophen-3-yl & 30 & 70 \\
$\mathbf{1 m}$ & 5-Methylfuran-2-yl & 30 & 88 \\
$\mathbf{l n}$ & 5-Bromofuran-2-yl & 30 & 81 \\
\hline
\end{tabular}

${ }^{\mathrm{a}}$ Isolated yield.

the FTIR spectra of the 1-[(1,3-thiazol-2-ylamino)methyl]-2naphthol derivatives. In the ${ }^{1} \mathrm{H}$ NMR spectra, $\mathrm{NH}$ proton signals were observed at $8.29-9.36 \mathrm{ppm}$. The $\mathrm{CH}$ protons which are next to Ar groups were observed at $5.96-7.01 \mathrm{ppm}$, and the $\mathrm{OH}$ protons appeared in the range $10.08-12.23 \mathrm{ppm}$. The mass spectra of all new compounds showed the expected molecular ion peak.

1,3-Oxazine derivatives were prepared by the ring-closure reactions of 1-[(1,3-thiazol-2-ylamino)methyl]-2-naphthols $\mathbf{l a}, \mathbf{1 c}, \mathbf{1 d}, \mathbf{1 k}, \mathbf{1 m}$, and $1 \mathbf{n}$ with formaldehyde under ultrasound irradiation at $50^{\circ} \mathrm{C}$ (Table 4 and Scheme 4) in THF solvent. 


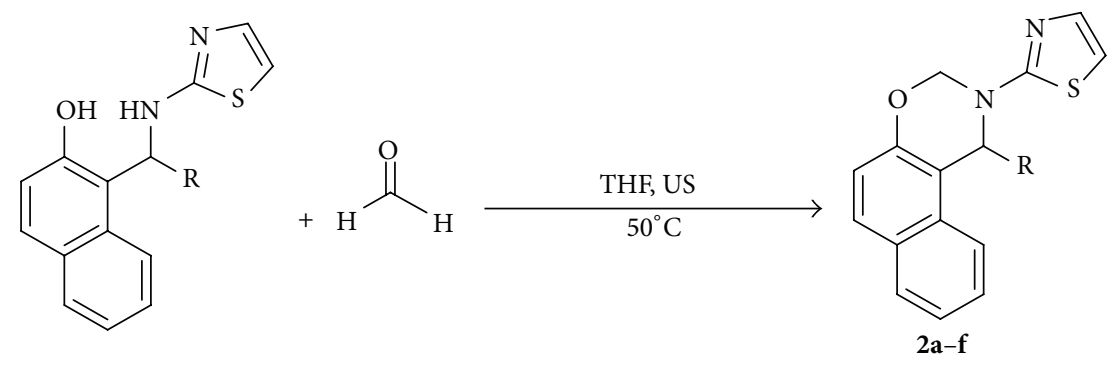

Scheme 4: Synthesis of naphthoxazines under US conditions.

TABLE 4: Synthesis of naphthoxazines (2a-f) (see Scheme 4).

\begin{tabular}{lccc}
\hline Product & R & Time (min) & Yield (\%) \\
\hline 2a & Phenyl & 120 & 53 \\
2b & 4-Bromophenyl & 120 & 36 \\
2c & 4-Isopropylphenyl & 120 & 69 \\
2d & Thiophen-2-yl & 120 & 64 \\
2e & 5-Methylfuran-2-yl & 120 & 65 \\
2f & 5-Bromofuran-2-yl & 120 & 57 \\
\hline
\end{tabular}

${ }^{a}$ Isolated yield.

The ring-closure reaction of $\mathbf{1 a}(1.00 \mathrm{mmol})$ with formaldehyde $(1.20 \mathrm{mmol})$ without a catalyst under conventional conditions at room temperature gave the desired product in $15 \mathrm{~h}$ with $40 \%$ yield. The best result was obtained by carrying out the reaction at $50^{\circ} \mathrm{C}$ under ultrasonic irradiation.

In the ${ }^{1} \mathrm{H}$ NMR spectra of 1,3-oxazine derivatives the $\mathrm{CH}_{2}$ protons which are between $\mathrm{N}$ and $\mathrm{O}$ atoms appeared in the ranges $4.98-5.37$ and 5.51-5.70 ppm. The $\mathrm{CH}$ protons which are near the Ar group were observed at 6.75-7.02 ppm.

\section{Conclusions}

In conclusion, we have described a greener, efficient, and practical method for the synthesis of 1-[(1,3-thiazol-2ylamino)methyl]-2-naphthols via a three-component onepot Mannich-type reaction of 2-aminothiazole, aromatic aldehydes, and 2-naphthol using commercially available, less expensive $(+)$-camphor-(10)-sulfonic acid $((+)$-CSA) as a metal-free catalyst under solvent-free ultrasound irradiation. The ring-closure reactions of the 1-[(1,3-thiazol-2ylamino)methyl]-2-naphthols with formaldehyde gave 1,3oxazine derivatives in moderate to good yields. The advantages of this synthesis are use of ultrasonic irradiation as energy source, nontoxic and economically viable catalyst, short reaction times, and simplified workup procedure.

\section{Competing Interests}

The authors declare that there are no competing interests regarding the publication of this paper.

\section{Acknowledgments}

This study was financially supported by Kırklareli University with the Project no. KLUBAP 015.

\section{References}

[1] A. Dömling, "Recent developments in isocyanide based multicomponent reactions in applied chemistry," Chemical Reviews, vol. 106, no. 1, pp. 17-89, 2006.

[2] J. Zhu, "Recent developments in the isonitrile-based multicomponent synthesis of heterocycles," European Journal of Organic Chemistry, no. 7, pp. 1133-1144, 2003.

[3] J. Zhu and H. Bienayme, Eds., Multicomponent Reactions, Wiley-VCH, Weinheim, Germany, 2005.

[4] J. D. Sunderhaus and S. F. Martin, "Applications of multicomponent reactions to the synthesis of diverse heterocyclic scaffolds," Chemistry-A European Journal, vol. 15, no. 6, pp. 1300-1308, 2009.

[5] A. Dömling and I. Ugi, "Multicomponent reactions with isocyanides," Angewandte Chemie-International Edition, vol. 39, no. 18, pp. 3169-3210, 2000.

[6] J. Zhu, Q. Wang, and M. X. Wang, Eds., Multicomponent Reactions in Organic Synthesis, Wiley-VCH, Weinheim, Germany, 2015.

[7] L. Pu and H.-B. Yu, "Catalytic asymmetric organozinc additions to carbonyl compounds," Chemical Reviews, vol. 101, no. 3, pp. 757-824, 2001.

[8] R. Müller, H. Goesmann, and H. Waldmann, "N,N-phthaloylamino acids as chiral auxiliaries in asymmetric Mannich-type reactions," Angewandte Chemie-International Edition, vol. 38, no. 1-2, pp. 184-187, 1999.

[9] M. Arend, B. Westermann, and N. Risch, "Modern variants of the Mannich reaction," Angewandte Chemie-International Edition, vol. 37, no. 8, pp. 1044-1070, 1998.

[10] S. G. Subramaniapillai, "Mannich reaction: a versatile and convenient approach to bioactive skeletons," Journal of Chemical Sciences, vol. 125, no. 3, pp. 467-482, 2013.

[11] G. Roman, "Mannich bases in medicinal chemistry and drug design," European Journal of Medicinal Chemistry, vol. 89, pp. 743-816, 2015.

[12] C. Chen, X. Zhu, Y. Wu et al., "5-Sulfosalicylic acid catalyzed direct Mannich reaction in pure water," Journal of Molecular Catalysis A: Chemical, vol. 395, no. 1, pp. 124-127, 2014.

[13] Z. Guan, J. Song, Y. Xue, D.-C. Yang, and Y.-H. He, "Enzymecatalyzed asymmetric Mannich reaction using acylase from Aspergillus melleus," Journal of Molecular Catalysis B: Enzymatic, vol. 111, pp. 16-20, 2015.

[14] W.-J. Hao, B. Jiang, S.-J. Tu et al., "A new mild base-catalyzed Mannich reaction of hetero-arylamines in water: highly efficient stereoselective synthesis of $\beta$-aminoketones under microwave heating," Organic \& Biomolecular Chemistry, vol. 7, no. 7, pp. 1410-1414, 2009. 
[15] U. C. Rajesh, R. Kholiya, V. Satya Pavan, and D. S. Rawat, "Catalyst-free, ethylene glycol promoted one-pot three component synthesis of 3-amino alkylated indoles via Mannich-type reaction," Tetrahedron Letters, vol. 55, no. 18, pp. 2977-2981, 2014.

[16] V. Kavala, C. Lin, C.-W. Kuo, H. Fang, and C.-F. Yao, "Iodine catalyzed one-pot synthesis of flavanone and tetrahydropyrimidine derivatives via Mannich type reaction," Tetrahedron, vol. 68, no. 4, pp. 1321-1329, 2012.

[17] C. Cimarelli, G. Palmieri, and E. Volpini, "A practical stereoselective synthesis of secondary and tertiary aminonaphthols: chiral ligands for enantioselective catalysts in the addition of diethylzinc to benzaldehyde," Tetrahedron: Asymmetry, vol. 13, no. 22, pp. 2417-2426, 2002.

[18] C. Cimarelli and G. Palmieri, "Synthesis of enantiopure 2(Aminoalkyl)phenol derivatives and their application as catalysts in stereoselective reactions," Chirality, vol. 21, no. 1, pp. 218232, 2009.

[19] I. Szatmári and F. Fülöp, "Syntheses, transformations and applications of aminonaphthol derivatives prepared via modified Mannich reactions," Tetrahedron, vol. 69, no. 4, pp. 1255-1278, 2013.

[20] E. Pelit and Z. Turgut, "Synthesis of enantiopure aminonaphthol derivatives under conventional/ultrasonic technique and their ring-closure reaction," Arabian Journal of Chemistry, 2014.

[21] A. Shaabani, A. Rahmati, and E. Farhangi, "Water promoted one-pot synthesis of $2^{\prime}$-aminobenzothiazolomethyl naphthols and 5-(2'-aminobenzothiazolomethyl)-6-hydroxyquinolines," Tetrahedron Letters, vol. 48, no. 41, pp. 7291-7294, 2007.

[22] A. Hosseinian and H. R. Shaterian, " $\mathrm{NaHSO}_{4} \cdot \mathrm{H}_{2} \mathrm{O}$ catalyzed multicomponent synthesis of 1-(Benzothiazolylamino) methyl2-naphthols under solvent-free conditions," Phosphorus, Sulfur and Silicon and the Related Elements, vol. 187, no. 9, pp. 10561063, 2012.

[23] Z. Li and X. Mao, "One-pot three-component solvent-free synthesis of 1-[(1,3-thiazol-2-ylamino)methyl]-2-naphthols," Heterocyclic Communications, vol. 17, no. 5-6, pp. 219-222, 2011.

[24] K. N. Venugopala, M. Krishnappa, S. K. Nayak et al., "Synthesis and antimosquito properties of 2,6-substituted benzo[d]thiazole and 2,4-substituted benzo[d]thiazole analogues against Anopheles arabiensis," European Journal of Medicinal Chemistry, vol. 65, no. 1, pp. 295-303, 2013.

[25] S. Javanshir, A. Ohanian, M. M. Heravi, M. R. Naimi-Jamal, and F. F. Bamoharram, "Ultrasound-promoted, rapid, green, one-pot synthesis of $2^{\prime}$-aminobenzothiazolomethylnaphthols via a multi-component reaction, catalyzed by heteropolyacid in aqueous media," Journal of Saudi Chemical Society, vol. 18, no. 5, pp. 502-506, 2014.

[26] G. Chaubet, L. T. Maillard, J. Martinez, and N. Masurier, "A tandem aza-FriedeleCrafts reaction/Hantzsch cyclization: a simple procedure to access polysubstituted 2-amino-1,3-thiazoles," Tetrahedron, vol. 67, no. 26, pp. 4897-4904, 2011.

[27] K. Kundu and S. K. Nayak, "( \pm )-Camphor-10-sulfonic acid catalyzed direct one-pot three-component Mannich type reaction of alkyl (hetero)aryl ketones under solvent-free conditions: application to the synthesis of aminochromans," RSC Advances, vol. 2, no. 2, pp. 480-486, 2012.

[28] A. Srivastava, S. Singh, and S. Samanta, “( \pm )-CSA catalyzed Friedel-Crafts alkylation of indoles with 3-ethoxycarbonyl-3hydoxyisoindolin-1-one: an easy access of 3-ethoxycarbonyl3 -indolylisoindolin-1-ones bearing a quaternary $\alpha$-amino acid moiety," Tetrahedron Letters, vol. 54, no. 11, pp. 1444-1448, 2013.
[29] X. Jiang, Z. Song, C. Xu, Q. Yao, and A. Zhang, “(D,L)-10-camphorsulfonic-acid-catalysed synthesis of diaryl-fused 2,8-dioxabicyclo[3.3.1]nonanes from 2-hydroxychalcones and naphthol derivatives," European Journal of Organic Chemistry, vol. 2014, no. 2, pp. 418-425, 2014.

[30] D. A. Engel, S. S. Lopez, and G. B. Dudley, "Lewis acid-catalyzed Meyer-Schuster reactions: methodology for the olefination of aldehydes and ketones," Tetrahedron, vol. 64, no. 29, pp. 69886996, 2008.

[31] N. Paul, M. Murugavel, S. Muthusubramanian, and D. Sriram, "Camphorsulfonic acid catalysed facile tandem double Friedlander annulation protocol for the synthesis of phenoxy linked bisquinoline derivatives and discovery of antitubercular agents," Bioorganic \& Medicinal Chemistry Letters, vol. 22, no. 4, pp. 1643-1648, 2012.

[32] P. V. Shinde, A. H. Kategaonkar, B. B. Shingate, and M. S. Shingare, "An organocatalyzed facile and rapid access to $\alpha$-hydroxy and $\alpha$-amino phosphonates under conventional/ultrasound technique," Tetrahedron Letters, vol. 52, no. 22, pp. 2889-2892, 2011.

[33] S. N. R. Mule, S. K. Battula, G. Velespula, D. R. Guda, and H. B. Ballikolla, "10-Camphorsulfonic acid ( $( \pm)$-CSA) catalyzed facile one-pot synthesis of a new class of 2,5-disubstituted 1,3,4oxadiazoles," RSC Advances, vol. 4, no. 102, pp. 58397-58403, 2014.

[34] B. K. Gorityala, S. Cai, J. Ma, and X.-W. Liu, "(S)-camphorsulfonic acid catalyzed highly stereoselective synthesis of pseudoglycosides," Bioorganic \& Medicinal Chemistry Letters, vol. 19, no. 11, pp. 3093-3095, 2009.

[35] S. S. Ali, S. Nasreen, M. Farooqui, S. H. Quadri, and S. Sultan, "Facile synthesis of coumarin under solvent-free condition," International Journal of Research in Pharmaceutical and Biomedical Sciences, vol. 2, no. 3, pp. 1229-1231, 2011.

[36] A. Srivastava, S. M. Mobin, and S. Samanta, “( \pm )-CSA catalyzed one-pot synthesis of 6,7-dihydrospiro[indole-3,1' -isoindoline]$2,3^{\prime}, 4(1 \mathrm{H}, 5 \mathrm{H})$-trione derivatives: easy access of spirooxindoles and ibophyllidine-like alkaloids," Tetrahedron Letters, vol. 55, no. 11, pp. 1863-1867, 2014.

[37] W.-M. Dai, J. Wu, K. C. Fong, M. Y. H. Lee, and C. W. Lau, "Regioselective synthesis of acyclic cis-enediynes via an acidcatalyzed rearrangement of 1,2-dialkynylallyl alcohols. Syntheses, computational calculations, and mechanism," Journal of Organic Chemistry, vol. 64, no. 14, pp. 5062-5082, 1999.

[38] R. M. Kellogg, J. W. Nieuwenhuijzen, K. Pouwer et al., "Dutch resolution: separation of enantiomers with families of resolving agents. A status report," Synthesis, no. 10, pp. 1626-1638, 2003.

[39] M. Vinatoru, E. Bartha, F. Badea, and J. L. Luche, "Sonochemical and thermal redox reactions of triphenylmethane and triphenylmethyl carbinol in nitrobenzene," Ultrasonics Sonochemistry, vol. 5, no. 1, pp. 27-31, 1998.

[40] H. Zeng, H. Li, and H. Shao, "One-pot three-component Mannich-type reactions using Sulfamic acid catalyst under ultrasound irradiation," Ultrasonics Sonochemistry, vol. 16, no. 6, pp. 758-762, 2009.

[41] J. Safari and Z. Zarnegar, "Ultrasound mediation for one-pot multi-component synthesis of amidoalkyl naphthols using new magnetic nanoparticles modified by ionic liquids," Ultrasonics Sonochemistry, vol. 21, no. 3, pp. 1132-1139, 2014.

[42] E. Pelit and Z. Turgut, "Three-component aza-Diels-Alder reactions using $\mathrm{Yb}(\mathrm{OTf})_{3}$ catalyst under conventional/ultrasonic techniques," Ultrasonics Sonochemistry, vol. 21, no. 4, pp. 1600$1607,2014$. 
[43] C. Dong, K. Sanjay, and M. Ackmez, Handbook on Applications of Ultrasound Sonochemistry for Sustainability, CRC Press, Taylor \& Francis Group, Boca Raton, Fla, USA, 2012.

[44] T. J. Mason and D. Peters, "Practical Sonochemistry," in Power Ultrasound Uses and Applications, Ellis Horwood, New York, NY, USA, 2002.

[45] T. J. Mason, "Sonochemistry and the environment—providing a 'green' link between chemistry, physics and engineering," Ultrasonics Sonochemistry, vol. 14, no. 4, pp. 476-483, 2007.

[46] J. L. Luche, Synthetic Organic Sonochemistry, Plenum Press, New York, NY, USA, 1998.

[47] J.-T. Li, S.-X. Wang, G.-F. Chen, and T.-S. Li, "Some applications of ultrasound irradiation in organic synthesis," Current Organic Synthesis, vol. 2, no. 3, pp. 415-436, 2005.

[48] R. Gallardo-Macias and K. Nakayama, "Tin(II) compounds as catalysts for the Kabachnik-fields reaction under solvent-free conditions: facile synthesis of $\alpha$-aminophosphonates," Synthesis, no. 1, pp. 57-62, 2010.

[49] M. Adib, E. Sheibani, M. Mostofi, K. Ghanbary, and H. R. Bijanzadeh, "Efficient highly diastereoselective synthesis of 1,8adihydro-7H-imidazo[2,1-b][1,3] oxazines," Tetrahedron, vol. 62, no. 14, pp. 3435-3438, 2006.

[50] T. Kurz, "Synthesis of novel pyrido[2,3-e][1,3] oxazines," Tetrahedron, vol. 61, no. 12, pp. 3091-3096, 2005.

[51] P. Zhang, E. A. Terefenko, A. Fensome, J. Wrobel, R. Winneker, and Z. Zhang, "Novel 6-aryl-1,4-dihydrobenzo[d] and oxazine2 -thiones as potent, selective, and orally active nonsteroidal progesterone receptor agonists," Bioorganic \& Medicial Chemistry Letters, vol. 13, no. 7, pp. 1313-1316, 2003.

[52] H. Van de Poel, G. Guilaumet, and M. C. Viaud-Massuard, "Synthesis of 6,7,8,9-tetrahydropyrido[2,3- $b]$ indolizine and 3,4dihydro- $2 H$-pyrido $\left[2^{\prime}, 3^{\prime}: 4,5\right]$ pyrrolo[2,1- $\left.b\right][1,3]$ oxazine derivatives as new melatonin receptor ligands," Tetrahedron Letters, vol. 43, no. 7, pp. 1205-1208, 2002.

[53] S. Wang, Y. Li, Y. Liu, A. Lu, and Q. You, "Novel hexacyclic camptothecin derivatives. Part 1. Synthesis and cytotoxicity of camptothecins with an A-ring fused 1,3-oxazine ring," Bioorganic \& Medicinal Chemistry Letters, vol. 18, no. 14, pp. 40954097, 2008.

[54] S.-H. Zhao, J. Berger, R. D. Clark et al., "3,4-Dihydro-2Hbenzo[1,4] oxazine derivatives as 5-HT6 receptor antagonists," Bioorganic \& Medicinal Chemistry Letters, vol. 17, no. 12, pp. 3504-3507, 2007.

[55] Y. Tabuchi, Y. Ando, H. Kanemura et al., "Preparation of novel (Z)-4-ylidenebenzo[b]furo[3,2-d][1,3] oxazines and their biological activity," Bioorganic \& Medicinal Chemistry, vol. 17, no. 11, pp. 3959-3967, 2009.

[56] N. Zanatta, A. M. C. Squizani, L. Fantinel et al., "Synthesis of $N$-substituted 6-trifluoromethyl-1,3-oxazinanes," Journal of the Brazilian Chemical Society, vol. 16, no. 6, pp. 1255-1261, 2005.

[57] J. N. Joyce, S. Presgraves, L. Renish et al., "Neuroprotective effects of the novel $\mathrm{D}_{3} / \mathrm{D}_{2}$ receptor agonist and antiparkinson agent, S32504, in vitro against 1-methyl-4-phenylpyridinium (MPP+) and in vivo against 1-methyl-4-phenyl-1,2,3,6tetrahydropyridine (MPTP): a comparison to ropinirole," Experimental Neurology, vol. 184, no. 1, pp. 393-407, 2003.

[58] F. A. J. Kerdesky, "A novel and efficient method for the conversion of a trans-hexahydronaphthoxazine to a cis-isomer using boron tribromide," Tetrahedron Letters, vol. 46, no. 10, pp. 17111712, 2005.
[59] C. Cimarelli, A. Mazzanti, G. Palmieri, and E. Volpini, "Solventfree asymmetric aminoalkylation of electron-rich aromatic compounds: stereoselective synthesis of aminoalkylnaphthols by crystallization-induced asymmetric transformation," Journal of Organic Chemistry, vol. 66, no. 14, pp. 4759-4765, 2001.

[60] M. Heydenreich, A. Koch, S. Klod, I. Szatmári, F. Fülöp, and E. Kleinpeter, "Synthesis and conformational analysis of naphth $\left[1^{\prime}, 2^{\prime}: 5,6\right][1,3]$ oxazino [3,2-c] [1,3] benzoxazine and naphth $\left[1^{\prime}, 2^{\prime}: 5,6\right][1,3]$ oxazino[3,4-c] $[1,3]$ benzoxazine derivatives," Tetrahedron, vol. 62, no. 48, pp. 11081-11089, 2006.

[61] R. Csütörtöki, I. Szatmári, A. Koch, M. Heydenreich, E. Kleinpeter, and F. Fülöp, "Synthesis and conformational analysis of new naphth[1,2-e][1,3] oxazino[3,4-c] quinazoline derivatives," Tetrahedron, vol. 67, no. 44, pp. 8564-8571, 2011.

[62] R. Csütörtöki, I. Szatmári, M. Heydenreich et al., "Novel piperidine-fused benzoxazino-and quinazolinonaphthoxazines-synthesis and conformational study," Tetrahedron, vol. 68, no. 31, pp. 6284-6288, 2012.

[63] Z. Turgut, E. Pelit, and A. Köycü, "Synthesis of New 1,3-Disubstituted-2,3-dihydro-1H-naphth[1,2e][1,3] oxazines," Molecules, vol. 12, no. 3, pp. 345-352, 2007.

[64] H. Cao, H.-F. Jiang, C.-R. Qi, W.-J. Yao, and H.-J. Chen, "Brønsted acid-promoted domino reactions: a novel one-pot three-component synthesis of 3,4,5-trisubstituted-3,6-dihydro2H-1,3-oxazines," Tetrahedron Letters, vol. 50, no. 11, pp. 12091214, 2009.

[65] V. D. Dhakane, S. S. Gholap, U. P. Deshmukh, H. V. Chavan, and B. P. Bandgar, "An efficient and green method for the synthesis of $[1,3]$ oxazine derivatives catalyzed by thiamine hydrochloride (VB1) in water," Comptes Rendus Chimie, vol. 17, no. 5, pp. 431436, 2014.

[66] T. Sun, Q. Cai, M. Li et al., "Facile diastereoselective synthesis of cis-perfluoroalkylated fused [1,3] oxazines from aromatic aldehydes, methyl perfluoroalk-2-ynoates and quinolines," Tetrahedron, vol. 71, no. 4, pp. 622-629, 2015.

[67] S. Fiorito, S. Genovese, V. A. Taddeo, and F. Epifano, "Microwave-assisted synthesis of coumarin-3-carboxylic acids under ytterbium triflate catalysis," Tetrahedron Letters, vol. 56, no. 19, pp. 2434-2436, 2015.

[68] A. A. Pereira, P. P. de Castro, A. C. de Mello, B. R. V. Ferreira, M. N. Eberlin, and G. W. Amarante, "Brønsted acid catalyzed azlactone ring opening by nucleophiles," Tetrahedron, vol. 70, no. 20, pp. 3271-3275, 2014.

[69] Y. Chen, W. Shan, M. Lei, and L. Hu, "Thiamine hydrochloride $\left(\mathrm{VB}_{1}\right)$ as an efficient promoter for the one-pot synthesis of 2,3dihydroquinazolin-4(1H)-ones," Tetrahedron Letters, vol. 53, no. 44, pp. 5923-5925, 2012.

[70] M. Vafaeezadeh and H. Alinezhad, "Brønsted acidic ionic liquids: green catalysts for essential organic reactions," Journal of Molecular Liquids, vol. 218, no. 1, pp. 95-105, 2016. 

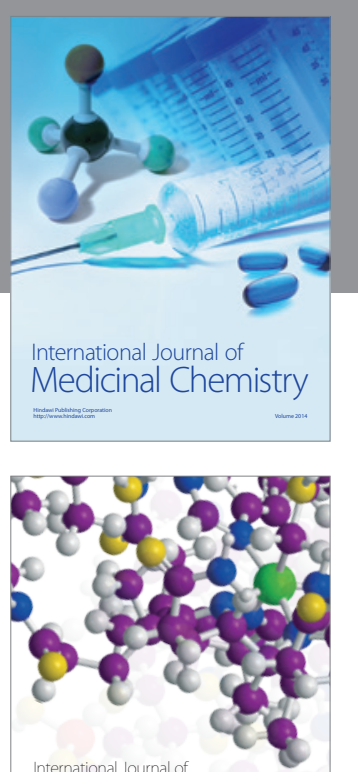

Carbohydrate Chemistry

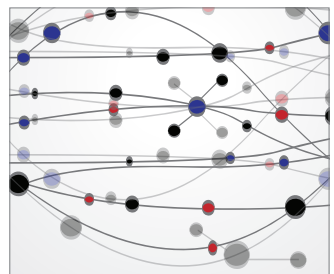

The Scientific World Journal
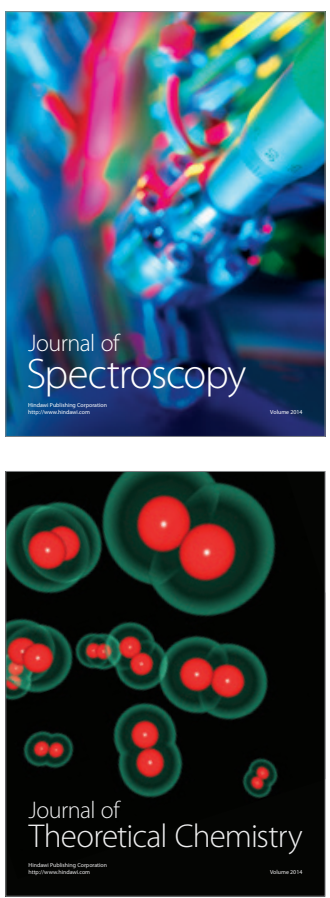
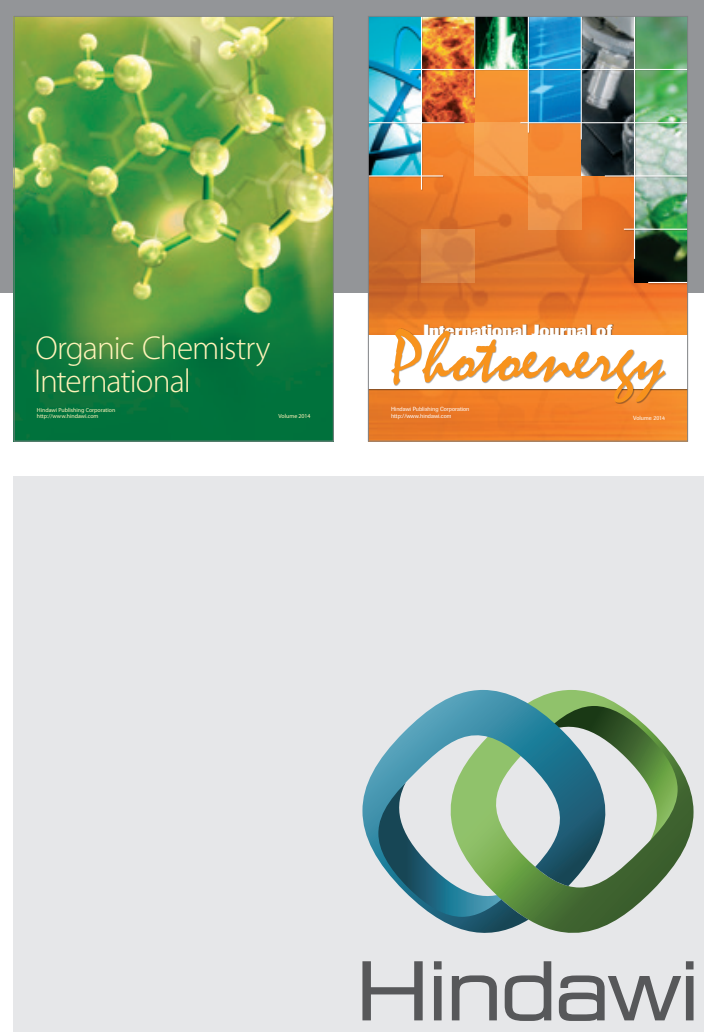

Submit your manuscripts at

http://www.hindawi.com

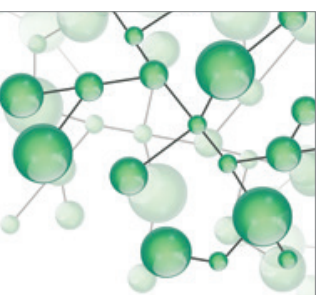

International Journal of

Inorganic Chemistry

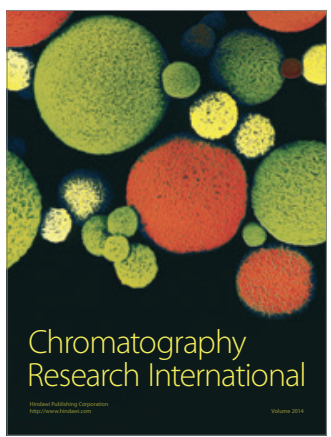

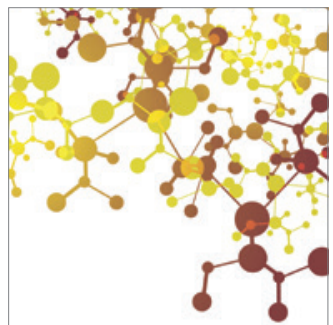

Applied Chemistry
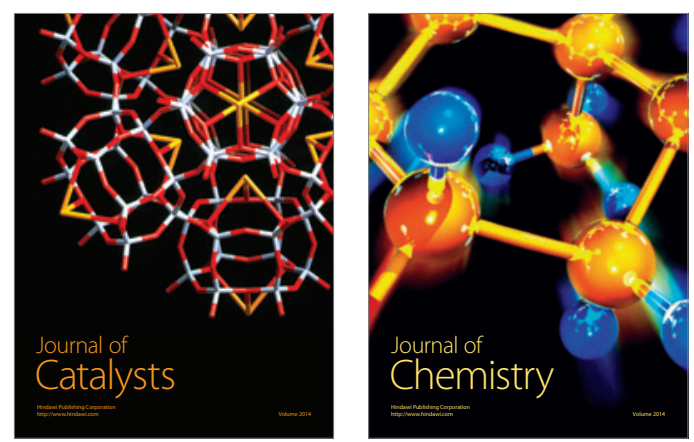
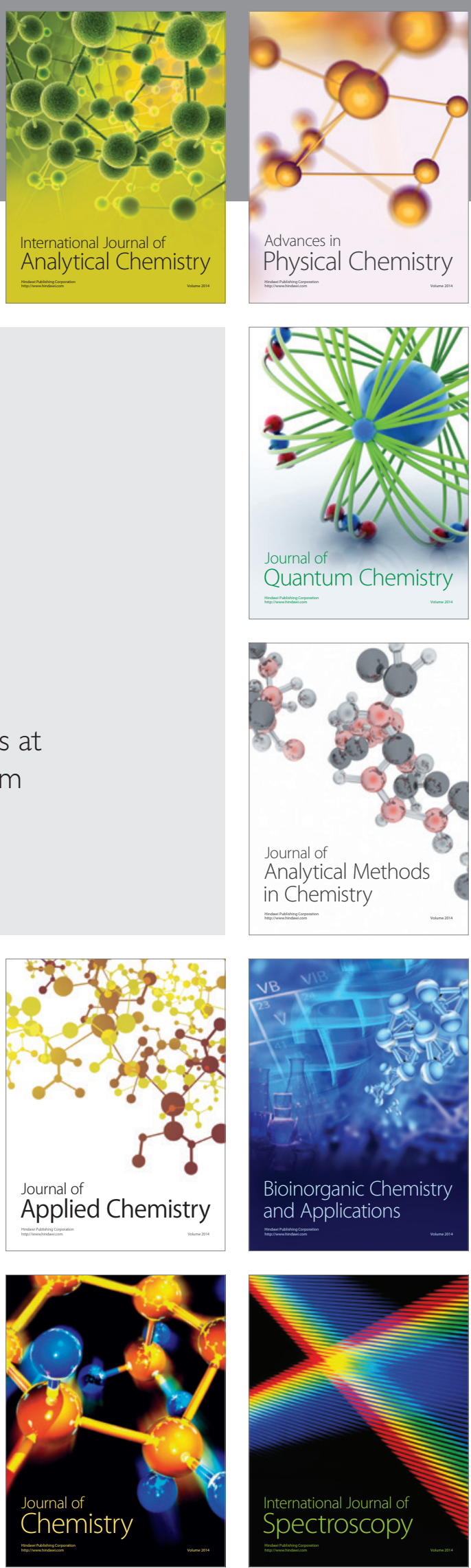